\title{
Conflict Identification among Usability Requirements Using Fuzzy Logic
}

\author{
Kanza Gulzar, Jun Sang, and Muhammad Ramzan
}

\begin{abstract}
Usability Engineering is one of the most exciting and evolving areas of the research in the domain of human-computer interaction (HCI) and an integral part of HCI. Significant investigation has been conducted on the associations and conflicts between quality aspects but not much of work has been carried out on the identification of conflicts among usability requirement attributes separately. In this paper we have proposed a framework to examine the usability attributes trade-offs, involved in software development. It identifies the conflicts among usability requirement attributes and quantifies these conflicts using fuzzy methodology. The use of fuzzy modeling to deal with vagueness and contradictions in usability factors and its sub-factors makes this study a valuable contribution on this subject.
\end{abstract}

Index Terms-Human computer Interaction (HCI), usability requirement (URs), conflict identification, fuzzy logic.

\section{INTRODUCTION}

I is well known that usability is one of the most significant factors which allows the users to communicate with system in affective manner and also represents an important component in the field of HCI. The ISO 9241-11 [1] defines usability as "the context to which a product can be used by specified users to achieve specified goals with effectiveness, efficiency and satisfaction in a specified context of use”. It is clear from the definition that usability has a set of attributes, prerequisite to start work on any software product. One thing to be noticed is that the set of attributes are not defined and are left on the thinking of the system evaluator. A subset of these definitions is summarized in Table I.

Considering the importance of Usability it is realized that it is wrong concept to add usability at the lateral stages of the software development process. To develop cost effective and usable system it is necessary to develop architecture that supports usability [2]. So, it is of great importance to gather and compile the usability requirements from all concerned and identify the inherent conflicts among them along with the low level attributes. The major problem encountered in this regard is the fact that the stakeholders, especially the users, do not have an idea about the usability requirements at an early stage. To identify and appropriately manage usability

Manuscript received June 27, 2016; revised December 25, 2016.

K. Gulzar is with School of Software Engineering, Chongqing University, Chongqing 401331, China, pursuing her $\mathrm{PhD}$., on leave from the Arid Agriculture University Rawalpindi Pakistan (e-mail: kanza@uaar.edu.pk, kaniyagi@hotmail.com).

J. Sang is with School of Software Engineering, Chongqing University, Chongqing 401331, China.

M. Ramzan was with University Institute of Information Technology, Arid Agriculture University Rawalpindi Pakistan now is working in College of Computing and Informatics, Saudi Electronic University, Riyadh, Saudi Arabia. requirement conflicts is a challenging task.

The Fuzzy Logic is a mathematical tool that facilitates to attain the goal with precise conclusion even using ambiguous rough, inexact, or omitted received information [3]. This idea can be helpful along with previous experience in making decisions about a certain situation. However, in general, the past experiences of usability requirements are rarely stored and utilized. Giving importance to previous knowledge, an organization with a better knowledge base of usability requirements factors and sub factors can effectively identify and resolve conflicts. So, there is, need of a frame-work which provides a systematic approach to identify conflicts among usability requirements by sorting out the correlation between different factors (attributes) of usability and conflicts among them and quantify these conflicts.

Next section presents the related work that is followed by Section III describing the proposed methodology. Section IV analyze and discuss the proposed framework while the last section concludes the paper with future work description.

\section{RELATED WORK}

Traditionally, requirements are classified into two broad categories, functional requirements and non-functional requirements. The best way to define software requirements is the desired working of the system along with all of its quality concerns. Quality concerns are also discussed in literature and the authors described usability as quality of use. For example for a graphical user interface it should be both usable and useful, means performing the said functions for which it is intended to develop with a certain level of accuracy.

The most difficult task is to decide what needs to be fabricated. Meeting all the requirements of a variety of stakeholders may not be possible all the time. Boehm [4] dealt with nonfunctional requirements at an abstract level and prioritized these requirements using perspective of the stakeholders. In a more recent publication, Jenny Preece [5] distinguished between two types of usability attributes: "usability goals" and "user experience". Identification of conflicts among requirements is a challenging task in software engineering [6]. Quality attributes and traceability techniques are helpful in this regard.

Egyed and Grunbacher [7] proposed another approach, that defines a method to control the growth and complexity of requirements. It helps developers to recognize requirements conflicts that can damage the NFRs. Xu et al proposed a method for determining nonfunctional requirement satisfaction by modelling partial state charts for design alternatives to satisfy the non-functional requirements [8]. 
A set of heuristics for meeting and later measuring the NFCs are also proposed. The relationship between software architecture an usability was first discussed in [9] by offering an architectural model that facilitate the designer to satisfy the ergonomic features. Chi Lin Liu [10] developed a process for defining non-functional requirement and detecting conflicts. This model defines the NF requirements by using value ontologies and for the detection of the non-functional requirement conflicts a set of rules is defined. In 2009, Sauro [11] described the strong correlations (between 0.44 and 0.60) between the standard usability aspects on the basis of data gathered from 90 usability tests.

TABLE I: THE USABILITY ATTRIBUTES DEFINED BY FORMER AND BOSH [2]

\begin{tabular}{|c|c|c|c|c|c|}
\hline $\begin{array}{l}\text { Shackel } \\
\text { (1991) }\end{array}$ & $\begin{array}{c}\text { Schneiderman } \\
\text { (1992) }\end{array}$ & $\begin{array}{l}\text { Nielson } \\
\text { (1993) }\end{array}$ & $\begin{array}{l}\text { Preece et al. } \\
\text { (1994) }\end{array}$ & $\begin{array}{c}\text { ISO } 9241-11 \\
(1998)\end{array}$ & $\begin{array}{l}\text { ISO } 9241-11 \\
(1998)\end{array}$ \\
\hline $\begin{array}{l}\text { Effectiveness } \\
\text { (speed) }\end{array}$ & $\begin{array}{c}\text { Speed of } \\
\text { performance }\end{array}$ & $\begin{array}{l}\text { Efficiency } \\
\text { of use }\end{array}$ & Throughput & Efficiency & $\begin{array}{l}\text { Efficiency } \\
\text { in use }\end{array}$ \\
\hline $\begin{array}{l}\text { Learnability Time } \\
\text { for learning }\end{array}$ & Time to learn & $\begin{array}{c}\text { Learnability } \\
\text { (Easy to learn) }\end{array}$ & $\begin{array}{c}\text { Learnability } \\
\text { (Easy to learn) }\end{array}$ & & Learnability \\
\hline $\begin{array}{c}\text { Learnability } \\
\text { retention }\end{array}$ & $\begin{array}{c}\text { Retention over } \\
\text { interval }\end{array}$ & Memorability & & & $\begin{array}{c}\text { Remember } \\
\text { ability }\end{array}$ \\
\hline $\begin{array}{l}\text { Effectiveness } \\
\text { error }\end{array}$ & $\begin{array}{l}\text { Rate of Errors } \\
\text { by user }\end{array}$ & Error & Throughput & & $\begin{array}{l}\text { Reliability } \\
\text { in use }\end{array}$ \\
\hline Attitude & $\begin{array}{l}\text { Subjective } \\
\text { satisfaction }\end{array}$ & Satisfaction & Attitude & $\begin{array}{c}\text { Satisfaction } \\
\text { (comfort ability and } \\
\text { acceptability) }\end{array}$ & $\begin{array}{c}\text { User } \\
\text { satisfaction }\end{array}$ \\
\hline
\end{tabular}

Co-authors of this work have been working for a long time to incorporate artificial intelligence in various areas of requirement engineering [12]. Now we focus on implementing intelligent frameworks for usability requirements. According to Dubey [13], the usability of a system is improved by combining the software engineering process with usability engineering in an object-oriented approach. They proposed an extended version of ISO-9241 usability model with fuzzy modeling approach. A conflict among the usability requirements can be seen while improving learn- ability (an attribute of usability), is to develop systems which can direct a user through step by step guidance [14]. However, in some cases, the functionality of the system can affect the overall efficiency in a negative way. Sometimes, easier to learn systems can be inefficient and harder to learn systems could be more efficient as shown in Fig. 1.

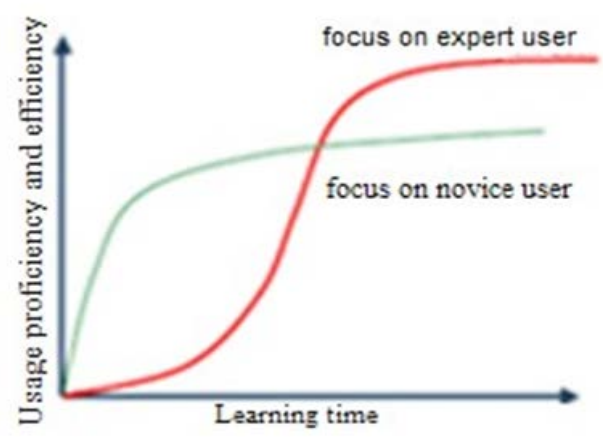

Fig. 1. Increased learn ability decreases efficiency [14].

In next section we proposed a framework to identify potential conflicts among usability requirement attributes early in the software or system development life cycle. The proposed framework examines the usability attribute tradeoffs involved in software development.

\section{FUZZY LOGIC APPROACH TO USABILITY REQUIREMENTS}

As discussed in the previous section, a variety of tech-niques have been proposed for prioritization of require-ments in general. An appropriate framework that could effectively identify conflicts among usability requirements and rank these conflicts, however, is something that has been missing. We proposed such a framework that can allow to assess the conflicts and quantify the severity of conflicts. An overview of the proposed framework is illustrated in Fig. 2.

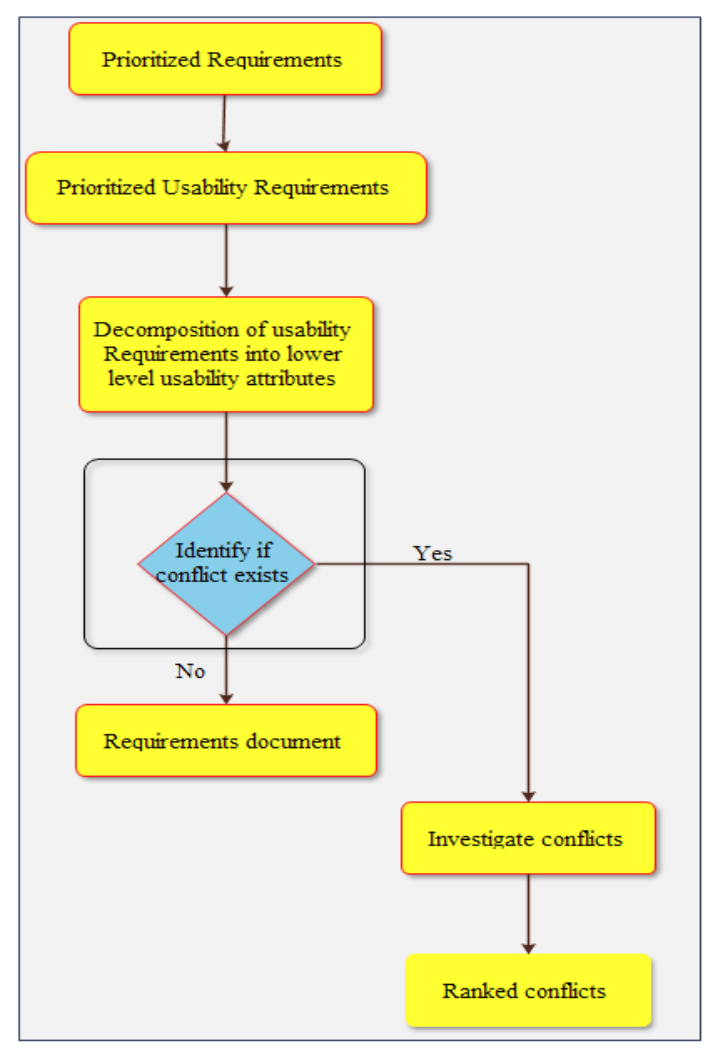

Fig. 2. Overview of the proposed framework usability.

Prioritization of requirements plays a very important role in the software development. Only the gathered requirements are the detailed explanation of user's needs. The Prioritized requirements will leads to better conflicts resolution and help in taking decisions related to trade- off among the gathered requirements. We will take the prioritized requirements from the users and the usability expert and domain expert will formulate and prioritize the usability requirements according to the feasibility of the 
project.

To limit the scope of the study to identify the conflicts among usability requirements (attributes / factors), we will use factors, described by Nielson [14]. In addition, we will also use the attribute Effectiveness defined by Shackel [9] and ISO that is generally assessed through different questioners and literature survey. These are: Learnability, Efficiency, Memorability, Errors, Satisfaction and Effectiveness.

There is a lot of research that provides strategies for good and usable systems [10], [14]. Such knowledge can be useful, so a repository / director will be used in proposed framework to detain knowledge depicting conflicts among usability requirements in various situations. In this repository, which we term as knowledge factory, usability attributes will be deduced by refining it into sub-factors and sub-sub-factors connecting them to executable mechanisms. For each attribute scheme identified, determine negative effects on other attributes that leads to rise attribute based conflicts among usability requirements using repository of knowledge. A concise view of the proposed repository is given in Fig. 3.

After identifying conflicts manually from the repository, we will formulate a table (See Table II) for assignment of fuzzy values to the factors for application of Mamdani [15] method to quantify the conflicts. It works on the basis of fuzzy reasoning and has the form of an if-then conditional statement.

\begin{tabular}{|c|c|c|c|c|c|c|}
\hline Rules & $\begin{array}{c}\text { USABILITY } \\
\text { ATTRIBUTE } 1\end{array}$ & $\begin{array}{c}\text { USABILITY } \\
\text { ATTRIBUTE } 2\end{array}$ & $\begin{array}{l}\text { USABILITY } \\
\text { ATTRIBUTE } 3\end{array}$ & $\begin{array}{c}\text { USABILITY } \\
\text { ATTRIBUTE } 4\end{array}$ & $\begin{array}{l}\text { USABILITY } \\
\text { ATTRIBUTE } 5\end{array}$ & Conflict \\
\hline 1 & High & High & High & High & High & Very Serious \\
\hline 2 & High & High & High & High & Medium & Very Serious \\
\hline 3 & High & High & High & Medium & Low & Serious \\
\hline 4 & High & High & High & Medium & High & Very Serious \\
\hline . & . & - & . & - & - & . \\
\hline - & . & . & . & - & . & . \\
\hline $\mathrm{N}$ & $\mathrm{N}$ & $\mathrm{N}$ & $\mathrm{N}$ & $\mathrm{N}$ & $\mathrm{N}$ & $\mathrm{N}$ \\
\hline
\end{tabular}

IF ((Condition I is True) AND (Condition II is True)) THEN (Result can be inferred) Zadeh in [3] presented fuzzy set theory to handle the problems having vague and indistinguishable facts. There is associated membership function in each members of a fuzzy set that handles indefinite information, such as near to 4 or below average. We will used triangular numbers. The most commonly used forms of fuzzy numbers are triangular and trapezoidal membership functions. The mapping between any two factors can be articulated by integer scores ranging from 1,2 , 3 representing 'Low', 'Moderate', and 'High' respectively. Fuzzy numbers will be translated by these crisp scores based on Table III.

TABLE III: THE CORRESPONDING LINGUISTIC TERMS AND FUZZY NUMBERS \begin{tabular}{|l|l|l|}
\hline Li nguistic terms & Influence score & Triangular Fuzzy Numbers \\
\hline
\end{tabular}

\begin{tabular}{|c|c|c|}
\hline Low & 1 & $(0.0, .0 .1,0.3)$ \\
\hline Moderate & 2 & $(0.2,0.4,0.6)$ \\
\hline High & 3 & $(0.5,0.7,0.9)$ \\
\hline
\end{tabular}

\section{ANALYSIS AND DISCUSSION}

In this section we present a qualitative analysis of strengths of proposed work. As it evidents from the structure of technique, this fuzzy based planning assessment approach will quantitatively identify the possible conflicts between usability requirements. These conflicts will be based on linguistic assessments of attributes of usability requirements gathered from all the stakeholders. After specifying the fuzzy values, the proposed frame-work will give the a solution that will lead to achieve a minimum defined level of usability because it contributes to identify quantitatively positive or negative correlation among different usability attributes (sub-factors and sub- sub-factors) such as Learnability and Efficiency (easy to use verses efficient to use). Currently, NFR framework is boosted for the URs and will be applied in a numeric way and identified conflicts will then be prioritized and ranked quantitatively. This will help in better assessment of factors leading to enhanced user experience.

Another major benefit we assume to achieve by introducing this technique is automation and intelligence. This is critical because manual assessment of required usability factors can lead to ambiguities which can potentially cause the project to fail. By introducing an automated and intelligent technique, we expect to achieve a standard which in the case of correct requirement elicitation will always result in correct identification of usability factors. This would also help in achieving efficiency as against the human based techniques; proposed intelligent and automated approach would give correct and optimal results in considerably short span of time. Another major achievement of proposed work would be in the form of less human resource requirement which would on one hand help in easy scheduling of projects and on the other hand would reduce project cost. 


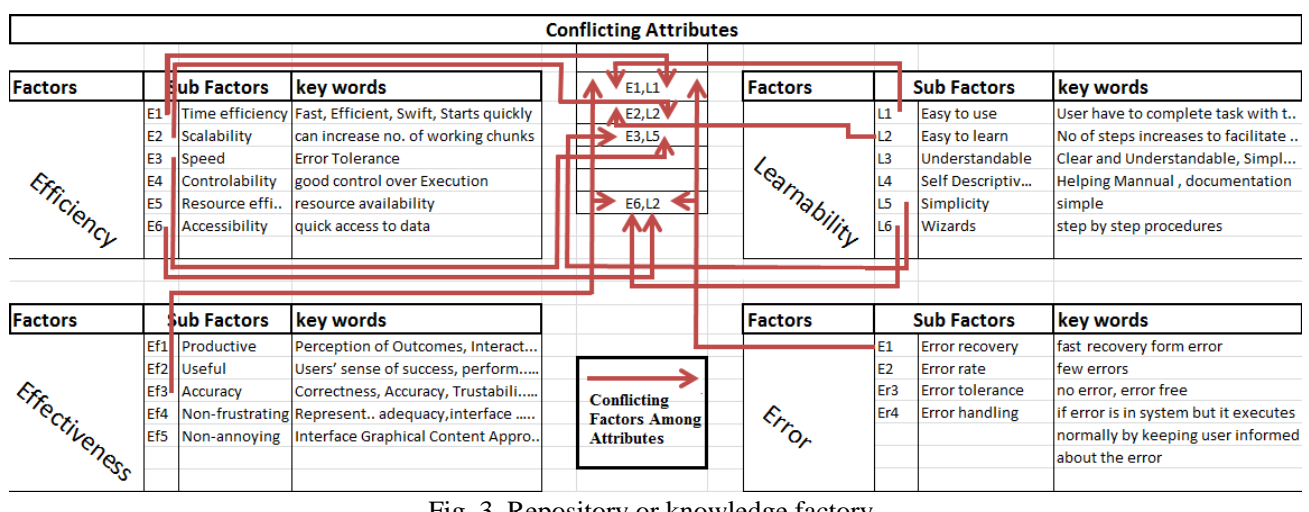

Fig. 3. Repository or knowledge factory.

In future the proposed framework will be evaluated by applying it on some scenarios, like here we have Electronic Health care System which should be enough usable to facilitate the doctors, nurses and other staff to perform their task smoothly without any hindrance and delay. As well as system should be pleasant to use with high level of accuracy and low error rate with in the specified time period. It is expected that by executing the proposed framework over such scenarios where the usability requirements have some contradictions and critical to meet, the proposed approach will be more flexible and better to identify and prioritize conflicts among usability requirement.

The point of concern is to be acquainted with the severity of identified conflicts that how much they are sever to affect the decision making. As in software development process different stakeholders are involved. They have different points of views that what is significant to them. This is important to any software project and establishes the need for making decisions for negotiation based on the priority of identified conflicts and the proposed framework allows the requirement analyst to work and take intuitive in more sophisticated and calculated way rather than thinking in verbal terms. It will improve the effectiveness of the usability requirement analysis by combining it with fuzzy methodology. It leads towards the accomplishment of obvious involvements of users in the system at user-interface level.

\section{CONCLUSION}

In this paper, we have presented an approach to identify the usability attributes and notify the conflicts in human computer interaction systems. Our specific emphasis is on such systems which are based on already existing systems as far as non-functional requirements are concerned. We have demonstrated the usefulness and superiority of pro-posed work through an empirical discussion and analysis. In future, we aim to validate the approach through its automation using fuzzy logic. We believe it would be very useful since Fuzzy models are the most appropriate for expressing imprecise and vague data such as human linguistic metaphors. The use of fuzzy logic for conflict identification among usability requirements is new in its category that combines the two domains. This work in future will incorporate the action of executing this frame work on large scale with multivariate concept.

\section{REFERENCES}

[1] International Organization for Standardization. "ISO. 9241-11, Ergonomic requirements for office work with visual display terminals (VDTs)," Part 11: Guidance on usability, Geneva Switzerland, 1998.

[2] E. Folmer and J. Bosch, "Architecting for usability: A survey," Journal of Systems and Software, vol. 70, no. 1-2, pp. 61-78, Feb. 2004.

[3] L. A. Zadeh, "From computing with numbers to computing with words-from manipulation of measurements to manipulation of perceptions," International Journal of Applied Mathematics and Computer Science, vol. 12 no. 3, pp. 307-324, 2002.

[4] B. Boehm and H. In, "Identifying quality-requirement conflicts," IEEE Software, vol. 13, no. 2, pp. 25-35, Mar. 1996.

[5] J. Preece, J., Y. Rogers et al., Interaction Design: Beyond Human-Computer Interaction, N.Y, USA: John Wiley And Sons, 2002.

[6] A. Kushniruk, "Evaluation in the design of health information systems: Application of approaches emerging from usability engineering," Computers in Biology and Medicine, vol. 32, no. 3, pp. 141-149, May 2002.

[7] A. Egyed and P. Grunbacher, "Identifying requirements conlicts and cooperation: How quality attributes and automated traceability can help, "IEEE Software, vol. 21, no. 6, pp. 50-58, Nov. 2004.

[8] L. Xu, S. Hendrickson, E. Hettwir, H. Ziv, A. Hoek, and D. Richardson, "Towards supporting the architectural design process through evaluation of design alternatives," in Proc. ISSTA workshop on Role of Software Architecture for Testing and Analysis, ACM New York, N.Y, USA, 2006.

[9] B. Shackel, "Usability - Context, framework, definition, design and evaluation," Interacting with Computers. vol. 21 no. 5-6, pp. 339-34 Dec. 2009

[10] L. Liu, Q. Liu, H. Chi, Z. Jin, and E. Yu, "Towards a service requirements modelling ontology based on agent knowledge and intentions," International Journal of Agent-Oriented Software Engineering, vol. 2, no. 3, pp. 324, 2008.

[11] J. Sauro and J. R. Lewis, "Correlations among prototypical usability metrics: evidence for the construct of usability." In Proc. 2009 Conference on Human Factors in Computing Systems ACM CHI, pp. 1609-1618, 2009.

[12] M. Ramzan, M. A. Jaffar, and A. A. Shahid, "Value based intelligent requirement prioritization (Virp): expert driven fuzzy logic based prioritization technique," International Journal of Innovative Computing, Information and Control, vol. 7, no. 3, pp. 1017-1038, 2011.

[13] S. K. Dubey, A. Rana, and A. Sharma, "Usability evaluation of object oriented software system using fuzzy logic approach," International Journal of Computer Applications, vol. 43, no. 19, pp. 1-6, Apr. 2012.

[14] J. Nielsen, Usability Engineering. Boston: Academic Press.

[15] E. H. Mamdani, "Application of fuzzy logic to approximate reasoning using linguistic synthesis," IEEE Transactions on Computers, vol. 26 no. 12, pp. 1182-1191, Dec. 1977. 


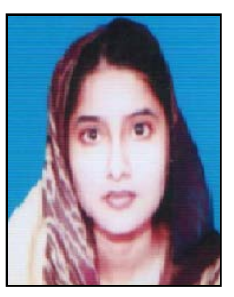

Kanza Gulzar was born in 1982 in Pakistan. She has done her MSc in computer science from University of Agriculture Faisalabad, Pakistan, in 2004. Her MS is in software engineering from Iqra University Islamabad, Pakistan. She is working as lecturer in PMAS Arid agriculture University Rawalpindi, Pakistan. She is currently pursuing her Ph.D in software engineering at Chongqing University, Chongqing, China. Her research interest includes software requirement engineering software quality assurance, knowledge management, decision support systems and HCI. She is a recipient of chinese government scholarship for Ph.D in software engineering from Chongqing University, P. R. China

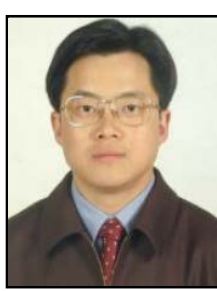

Jun Sang was born in Chongqing, China. He graduated from Shanghai Jiaotong University, Shanghai, China, and Chongqing University, Chongqing, China in 1990 and 1993, and received B.S. and M.E., respectively. He received the Ph.D degree in computer science in 2005 from Chongqing University. He is now a professor with School of software engineering at Chongqing University. His research interests include software engineering, digital image watermarking, information security, and image processing

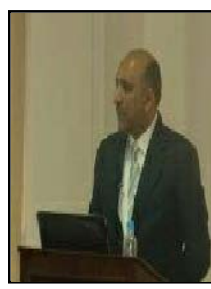

Muhammad Ramzan was born in Pakistan. He did his Ph.D from National University of Computer and Emerging Sciences in 2011. He is currently serving at College of Computing and Informatics. His areas of interest include software engineering, artificial intelligence and human computer interaction. He is author of several journal and conference publications. His work on intelligent requirement prioritization is widely read and cited. He is currently working in College of Computing and Informatics, Saudi Electronic University, Riyadh, Saudi Arabia. 